\title{
Bethe Ansatz for Quantum Strings
}

\author{
Gleb Arutyunov ${ }^{1, \dagger}$, Sergey Frolov ${ }^{2,1, \dagger}$ and Matthias Staudacher ${ }^{1}$ \\ ${ }^{1}$ Max-Planck-Institut für Gravitationsphysik, Albert-Einstein-Institut \\ Am Mühlenberg 1, D-14476 Potsdam, Germany \\ ${ }^{2}$ Department of Applied Mathematics, \\ SUNY Institute of Technology, \\ P.O. Box 3050, Utica, NY 13504-3050 \\ Email: agleb@aei.mpg.de; frolovs@sunyit.edu; matthias@aei.mpg.de
}

\begin{abstract}
We propose Bethe equations for the diagonalization of the Hamiltonian of quantum strings on $A d S_{5} \times S^{5}$ at large string tension and restricted to certain large charge states from a closed $\mathfrak{s u}(2)$ subsector. The ansatz differs from the recently proposed all-loop gauge theory asymptotic Bethe ansatz by additional factorized scattering terms for the local excitations. We also show that our ansatz quantitatively reproduces everything that is currently known about the string spectrum of these states. Firstly, by construction, we recover the integral Bethe equations describing semiclassical spinning strings. Secondly, we explain how to derive the $1 / \mathrm{J}$ energy corrections of $M$-impurity BMN states, provide explicit, general formulae for both distinct and confluent mode numbers, and compare to asymptotic gauge theory. In the special cases $M=2,3$ we reproduce the results of direct quantization of Callan et al. Lastly, at large string tension and relatively small charge we recover the famous $2 \sqrt[4]{n^{2} \lambda}$ asymptotics of massive string modes at level $n$. Remarkably, this behavior is entirely determined by the novel scattering terms. This is qualitatively consistent with the conjecture that these terms occur due to wrapping effects in gauge theory. Our finding does not in itself cure the disagreements between gauge and string theory, but leads us to speculate about the structure of an interpolating Bethe ansatz for the AdS/CFT system at finite coupling and charge.
\end{abstract}

$\dagger$ Also at Steklov Mathematical Institute, Moscow.

KEYwORDs: AdS-CFT Correspondence; Duality in Gauge Field Theories. 


\section{Contents}

1. Introduction 1

2. Bethe Ansatz for Quantum Strings 4

2.1 The Proposal

2.2 Heuristic Derivation 6

3. Near-BMN Limit 8

3.1 Separated Mode Numbers 8

3.2 Confluent Mode Numbers 11

4. Strong Coupling Limit 13

5. Wrapping Speculations 16

6. Summary and Conclusions 18

\section{Introduction}

Recently remarkable progress in our understanding of the gauge/string duality [1] was achieved. It was initiated by Berenstein, Maldacena and Nastase (BMN) [2], who proposed a way to match energies of certain string states with perturbative scaling dimensions of dual SYM operators.

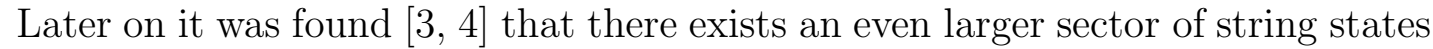
on $A d S_{5} \times S^{5}$ which a priori permits a direct comparison with perturbative gauge theory. Indeed a certain region of the quantum string spectrum can be well approximated by classical string solutions describing highly energetic strings. In most cases, however, comparison of the energy of such a string with a perturbative scaling dimension of the dual SYM operator is impossible since the string energy generically turns out to be non-analytic in the 't Hooft coupling constant $\lambda$. Fortunately, there exist solitonic solutions corresponding to classical multi-spin strings rapidly rotating in $S^{5}$ whose energies admit an expansion in integer powers of the effective coupling constant $\lambda / L^{2}$, where $L$ is the large, total spin on $S^{5}$. This offers the opportunity to directly compare the energies of spinning strings with perturbative scaling dimensions of gauge theory operators. Let us stress, however, that the comparison is not 
guaranteed to be successful, as the coupling $\lambda$ is nevertheless large in string theory, while it is small in perturbative gauge theory.

The simplest configurations are folded and circular rigid strings and the corresponding solutions can be obtained from an integrable system of the Neumann type [5] which is a finite-dimensional reduction of the classical string sigma-model on $A d S_{5} \times S^{5}$.

In a parallel development, related recent advances in gauge theory came to be after the important observation that planar, conformal $\mathcal{N}=4 \mathrm{SYM}$ is an integrable system in the one-loop approximation [6, 7] and, very likely, at higher loops as well [8]. This extends and generalizes in a beautiful way the previously observed integrable structures of one-loop QCD [9], which also happens to be conformal. Integrability is indispensable for resolving the complicated mixing problem in order to find the anomalous dimensions of conformal operators. The main new tool it provides is the Bethe ansatz, which reduces the spectral problem to the solution of a system of finitely many non-linear equations. For a closed $\mathfrak{s u}(2)$ subsector of operators the one-loop Bethe ansatz of [6] was extended to three loops in [10], using the threeloop integrable structures of [8]. Very recently an all-loop ansatz (under certain restrictions to be discussed below) was proposed in 11.

The Bethe ansatz allowed one to perform very non-trivial comparisons of gauge and string theory predictions as first shown in [12] in various special cases such as the above folded and circular rigid strings. Not only the energies but also the eigenvalues of higher charges agree at leading one-loop order [13], indicating that the integrable structures on both sides of the correspondence are closely related. Furthermore, it proved possible to exploit the classical integrability of the string sigma model and to derive a Bethe equation describing its semi-classical solutions for the simplest case of strings moving in $R \times S^{3}$ in full generality [14]. This led to perfect agreement between string and gauge theory structures up to two-loop order of perturbation theory. Further important work on the "spinning limit" of AdS/CFT was performed in 15, 16, 17], and other relevant aspects of the gauge/string duality have been studied in [18.

Starting at three-loop order this matching pattern breaks down both for spinning strings [10, 13] and strings in the near plane-wave background [19, 20]. One possible explanation was suggested in [10], and refined in [11]. Even though the effective coupling constant $\lambda / L^{2}$ is small the 't Hooft coupling $\lambda$ must be large for semiclassical spinning strings, as well as for strings in the near pp-wave background. Thus, a fully reliable comparison with gauge theory would require the complete summation of the gauge theoretic perturbative expansion, i.e. the knowledge of the spectrum of the allloop dilatation operator. In the spin chain picture the dilatation operator is identified with the Hamiltonian of a long-range spin chain. In particular, in the closed $\mathfrak{s u}(2)$ subsector the three-loop dilatation operator coincides [10] with the Hamiltonian of the integrable Inozemtsev spin chain [21]. At four loops the Inozemtsev chain violates 
BMN scaling, and, therefore, precludes perturbative comparison with string theory. Quite remarkably, another long-range spin chain exists which is different from the one of Inozemtsev starting at four loops, does not violate BMN scaling, and leads to the string-theoretic BMN energy formula [11]. Even though the explicit form of the all-loop Hamiltonian of this novel spin chain is currently unknown, the associated Bethe equations as well as the expressions for the eigenvalues of all higher conserved charges have been proposed in [1].

One intriguing result of [11], which will play a key role in this paper, was that the functional dependence of the individual charges of the elementary excitations (termed charge densities in [1]) on the excitation momenta agrees in the thermodynamic limit to all loop orders when one compares the (conjectured) gauge and (known) string theory expressions in the $\mathfrak{s u}(2)$ subsector. What is different is the distribution of excitation momenta. This may be traced back to the fact that the respective Bethe equations, whose solution precisely fixes the excitation momenta, differ by a certain term starting at three loop order.

As was discussed in [11] this difference might have two possible explanations. The first, somewhat disappointing one would be that the AdS/CFT correspondence is only approximate rather than exact. A second, more exciting one would be that non-perturbative effects have to be taken into account. In particular it was argued in [11] that these might arise after the inclusion of so-called wrapping interactions into the gauge theory computations. And indeed, the currently proposed Bethe ansatz of [11] is asymptotic in the sense that it correctly yields the gauge theory scaling dimensions only as long as the range of the spin chain interaction does not exceed the length of the spin chain. Unfortunately it is presently quite unclear how to properly account for the wrapping interactions on the gauge side.

The long-range Bethe equations of [11] and the classical Bethe equations of [14] conceptually differ in another important respect. The former are discrete equations applicable to a finite number of excitations (magnons) and at finite length (modulo the just explained restriction of asymptoticity). The latter are continuum ("thermodynamic") equations which assume a macroscopically large length and a large number of excitations. It is very natural to ask for a discrete version of the string Bethe equations. These should then properly describe the quantum corrections of the string sigma model. If we assume that integrability survives quantization on the string side these discrete equations should certainly exist. In this paper we will take inspiration from the spin chain equations to propose precisely such a set of equations engineered to account for the leading quantum corrections for strings on $A d S_{5} \times S^{5}$.

Our discretization reduces, by construction, in the thermodynamic limit to the integral Bethe equations describing classical spinning strings [14, 11]. We then use these equations to compute the leading finite length correction to the two- and threeimpurity BMN operators and find remarkable agreement with the results of [19] and [20]. We are also able to treat the multi-impurity problem in generality, which seems 
to be hardly possible in the framework of [19, 20]. In fact, our Bethe equations diagonalize the quantum string Hamiltonian to leading order in $1 / J$. We then turn to the study of the strong coupling limit. We use our equations to show that conformal dimensions of gauge theory operators dual to massive string modes at level $n$ exhibit the famous $2 \sqrt[4]{n^{2} \lambda}$ asymptotics. Remarkably, this behavior is completely due to the terms absent in the asymptotic gauge ansatz. While this fact of course does not prove the wrapping scenario, it is certainly fully consistent with it. In summary, the Bethe equations we propose are compatible with our current understanding of the spectrum of string theory on $A d S_{5} \times S^{5}$.

Our proposal is not yet complete as it is likely to need further refinement in order to include higher quantum string effects. However, we do speculate on a possible general form of the full quantum Bethe ansatz at the end of this paper. It is based on the idea that the correct ansatz should, if AdS/CFT is correct, interpolate between the string ansatz at large string tension $\sqrt{\lambda}$ and the gauge ansatz at small 't Hooft coupling $\lambda$. It would be important to find further evidence in its favor.

\section{Bethe Ansatz for Quantum Strings}

\subsection{The Proposal}

As was discussed in the introduction the classical sigma-model describing strings on $A d S_{5} \times S^{5}$ is an integrable system. One may hope that integrability is also preserved on the quantum level. In the $\mathfrak{s u}(2)$ subsector of string states with two spins, $M$ and $L-M$, the spectrum is encoded in a set of integral equations of Bethe type [14]. Thus, it is natural to expect that the quantum spectrum should be also described by a system of Bethe equations. These equations should diagonalize the quantum string Hamiltonian. Unfortunately the exact quantization of string theory is beyond our present reach. However, one can try to make an educated guess for such a system of equations, using our knowledge of the Bethe equations describing classical spinning strings and the asymptotic Bethe equations conjectured for the perturbative gauge theory.

The Bethe equations we propose for describing the leading quantum effects for strings in the $\mathfrak{s u}(2)$ sector have the following form

$$
\exp \left(i L p_{k}\right)=\prod_{\substack{j=1 \\ j \neq k}}^{M} S\left(p_{k}, p_{j}\right), \quad \sum_{k=1}^{M} p_{k}=0
$$

where the matrix $S\left(p_{k}, p_{j}\right)$ describing the pairwise scattering of local excitations with 
momenta $p_{k}$ is given by

$$
\begin{aligned}
S\left(p_{k}, p_{j}\right) & =\frac{\varphi\left(p_{k}\right)-\varphi\left(p_{j}\right)+i}{\varphi\left(p_{k}\right)-\varphi\left(p_{j}\right)-i} \times \\
& \times \exp \left(2 i \sum_{r=0}^{\infty}\left(\frac{g^{2}}{2}\right)^{r+2}\left(\mathbf{q}_{r+2}\left(p_{k}\right) \mathbf{q}_{r+3}\left(p_{j}\right)-\mathbf{q}_{r+3}\left(p_{k}\right) \mathbf{q}_{r+2}\left(p_{j}\right)\right)\right) .
\end{aligned}
$$

Here $M$ is the total number of local excitations and the phase function $\varphi(p)$ is

$$
\varphi(p)=\frac{1}{2} \cot \left(\frac{1}{2} p\right) \sqrt{1+8 g^{2} \sin ^{2}\left(\frac{1}{2} p\right)}
$$

where the coupling constant $g$ is related to the 't Hooft coupling $\lambda$ as

$$
g^{2}=\frac{\lambda}{8 \pi^{2}}
$$

The functions $\mathbf{q}_{r}(p)$ (charges) are given by

$$
\mathbf{q}_{r}(p)=g^{-r+1} \frac{2 \sin \left(\frac{1}{2}(r-1) p\right)}{r-1}\left(\frac{\sqrt{1+8 g^{2} \sin ^{2}\left(\frac{1}{2} p\right)}-1}{2 g \sin \left(\frac{1}{2} p\right)}\right)^{r-1}
$$

In particular, the first charge $\mathbf{q}_{1}(p)$ is the momentum $p$ and the second one $\mathbf{q}_{2}(p)$ is the energy of a single excitation

$$
\mathbf{q}_{2}(p)=\frac{1}{g^{2}}\left(\sqrt{1+8 g^{2} \sin ^{2}\left(\frac{1}{2} p\right)}-1\right) .
$$

In the sequel we will need the total charge of the $M$ excitations given by the sum of individual charges

$$
\mathbf{Q}_{r}=\sum_{k=1}^{M} \mathbf{q}_{r}\left(p_{k}\right)
$$

We expect this model to reproduce the quantum spectrum of string states with $L$ and $g$ large. The energy of string states in the global AdS coordinates is proposed to be

$$
\mathbf{E}(g)=L+g^{2} \mathbf{Q}_{2}
$$

The phase function $\varphi(p)$ and the charges $\mathbf{q}_{r}(p)$ first appeared in [11] where an all loop Bethe ansatz diagonalizing the dilatation operator in the $\mathfrak{s u}(2)$ subsector of $\mathcal{N}=4$ SYM theory in the asymptotic limit $L \rightarrow \infty$ was proposed. In fact our equations (2.1) differ from the asymptotic Bethe ansatz of [11] by the exponential term in the scattering matrix $S\left(p_{k}, p_{j}\right)$. Note that in the spin chain picture of [11] $L$ is identified with the length of the long-range spin chain, while $M$ is the number 
of magnons. The dilatation operator is related to the second charge $\mathbf{Q}_{2}$ in the same way as in eq.(2.7)

$$
\mathbf{D}(g)=L+g^{2} \mathbf{Q}_{2}
$$

Our proposal for the quantum Bethe ansatz is inspired by the observation of [11] that in the thermodynamic limit elementary excitations of gauge and string theory coincide. This motivates us to modify only the scattering matrix but to keep the same charges and phase function as in the gauge theory asymptotic Bethe ansatz. According to the AdS/CFT correspondence the string energy is equal to the scaling dimension of the dual gauge theory operator. In spite of the fact that we change the Bethe equations we still use the same relation between the energy/scaling dimension and the second conserved charge as in the asymptotic Bethe ansatz, i.e., as in perturbative gauge theory.

\subsection{Heuristic Derivation}

Here we present heuristic arguments motivating our proposal. We start from the integral Bethe equation which describes the classical spinning strings in the $\mathfrak{s u}(2)$ subsector [14]. As was shown in 11] the integral Bethe equations of [14 can be written as follows

$$
\begin{array}{r}
f_{\mathbf{C}} \frac{d \varphi^{\prime} \rho_{\mathrm{S}}\left(\varphi^{\prime}\right)}{\varphi-\varphi^{\prime}}=\frac{1}{2} \frac{1}{\sqrt{\varphi^{2}-4 \omega^{2}}}+\pi n_{\nu}+2 \omega^{2} \int_{\mathbf{C}} \frac{d \varphi^{\prime} \rho_{\mathrm{S}}\left(\varphi^{\prime}\right)}{\sqrt{\varphi^{2}-4 \omega^{2}} \sqrt{\varphi^{\prime 2}-4 \omega^{2}}} \times \\
\times \frac{\varphi-\sqrt{\varphi^{2}-4 \omega^{2}}-\varphi^{\prime}+\sqrt{\varphi^{\prime 2}-4 \omega^{2}}}{\left(\varphi+\sqrt{\varphi^{2}-4 \omega^{2}}\right)\left(\varphi^{\prime}+\sqrt{\varphi^{\prime 2}-4 \omega^{2}}\right)-4 \omega^{2}}
\end{array}
$$

where $n_{\nu}$ are winding numbers, and $\rho_{\mathrm{s}}(\varphi)$ is the spectral density of a finite-gap solution of the string sigma-model. The spectral density has a support on a union $\mathbf{C}$ of smooth contours in the complex $\varphi(p)$-plane and normalized as

$$
\int_{\mathbf{C}} d \varphi \rho_{\mathrm{s}}(\varphi)=\frac{M}{L}=\alpha
$$

In eq. (2.9) the effective coupling constant $\omega$ is defined as

$$
\omega^{2}=\frac{g^{2}}{2 L^{2}}
$$

Recall that the last term on the r.h.s. of eq.(2.9) is absent in the conjectured asymptotic gauge theory ansatz. Expanding the denominators of the last term in eq.(2.9) 
in a geometric series, we can rewrite this equation in the form

$$
\begin{aligned}
& f_{\mathbf{C}} \frac{d \varphi^{\prime} \rho_{\mathrm{S}}\left(\varphi^{\prime}\right)}{\varphi-\varphi^{\prime}}=\frac{1}{2} \frac{1}{\sqrt{\varphi^{2}-4 \omega^{2}}}+\pi n_{\nu}+\sum_{r=0}^{\infty} \omega^{2 r+4} \int_{\mathbf{C}} \frac{d \varphi^{\prime} \rho_{\mathrm{S}}\left(\varphi^{\prime}\right)}{\sqrt{\varphi^{2}-4 \omega^{2}} \sqrt{\varphi^{\prime 2}-4 \omega^{2}}} \times \\
& \times\left(\frac{1}{\left(\frac{1}{2} \varphi+\frac{1}{2} \sqrt{\varphi^{2}-4 \omega^{2}}\right)^{r+2}\left(\frac{1}{2} \varphi^{\prime}+\frac{1}{2} \sqrt{\varphi^{\prime 2}-4 \omega^{2}}\right)^{r+1}}-\right. \\
&\left.\frac{1}{\left(\frac{1}{2} \varphi+\frac{1}{2} \sqrt{\varphi^{2}-4 \omega^{2}}\right)^{r+1}\left(\frac{1}{2} \varphi^{\prime}+\frac{1}{2} \sqrt{\varphi^{\prime 2}-4 \omega^{2}}\right)^{r+2}}\right)
\end{aligned}
$$

According to [11] the commuting charge densities $\mathbf{q}_{r}(\phi)$ of the string sigma-model have the form

$$
\mathbf{q}_{r}(\varphi)=\frac{1}{\sqrt{\varphi^{2}-4 \omega^{2}}} \frac{1}{\left(\frac{1}{2} \varphi+\frac{1}{2} \sqrt{\varphi^{2}-4 \omega^{2}}\right)^{r-1}},
$$

where in particular $p(\varphi)=\mathbf{q}_{1}(\varphi)$. In terms of the spectral density the total commuting charges take the form

$$
\mathbf{Q}_{r}=\int_{\mathbf{C}} d \varphi \rho_{\mathrm{s}}(\varphi) \mathbf{q}_{r}(\varphi)
$$

Taking into account the expression for the charges we see that eqs.(2.11) can be cast into the following form

$$
f_{\mathbf{C}} \frac{d \varphi^{\prime} \rho_{\mathrm{s}}\left(\varphi^{\prime}\right)}{\varphi-\varphi^{\prime}}=\pi n_{\nu}+\frac{1}{2} p(\varphi)+\sum_{r=0}^{\infty} \omega^{2 r+4}\left(\mathbf{q}_{r+3}(\varphi) \mathbf{Q}_{r+2}-\mathbf{q}_{r+2}(\varphi) \mathbf{Q}_{r+3}\right)
$$

Now we note that this integral equation arises in the thermodynamic limit from the following discrete set of equations

$$
\begin{aligned}
\exp \left(i L p_{k}+2 i \sum_{r=0}^{\infty}\left(\frac{g^{2}}{2}\right)^{r+2}\left(\mathbf{q}_{r+3}\left(p_{k}\right) \mathbf{Q}_{r+2}-\mathbf{q}_{r+2}\left(p_{k}\right) \mathbf{Q}_{r+3}\right)\right) & = \\
= & \prod_{\substack{j=1 \\
j \neq k}}^{M} \frac{\varphi\left(p_{k}\right)-\varphi\left(p_{j}\right)+i}{\varphi\left(p_{k}\right)-\varphi\left(p_{j}\right)-i},
\end{aligned}
$$

where the charges $\mathbf{q}_{r}(p), \mathbf{Q}_{r}$ and the phase function $\varphi(p)$ are given by eqs.(2.4), (2.6) and (2.3). Indeed, in the thermodynamic limit $M$ and $L$ go to infinity with the ratio $\alpha=\frac{M}{L}$ held fixed. In this limit momentum $p_{k}$, charges $\mathbf{q}_{r}\left(p_{k}\right)$ and $\mathbf{Q}_{r}$ scale as $p_{k} \rightarrow p_{k} / L, \mathbf{q}_{r}\left(p_{k}\right) \rightarrow L^{-r} \mathbf{q}_{r}\left(p_{k}\right)$ and $\mathbf{Q}_{r} \rightarrow L^{-r+1} \mathbf{Q}_{r}$ respectively. The rescaled phase function $\varphi \rightarrow \varphi / L$ acquires the form

$$
\varphi(p)=\frac{1}{p} \sqrt{1+4 \omega^{2} p^{2}}
$$


To compare with the integral Bethe equation (2.14) it is useful to express $p$ and all other charges as functions of $\varphi$. Then one can see that the rescaled charges $\mathbf{q}_{r}(\varphi)$ coincide with (2.12). Finally to obtain the integral Bethe equations we introduce the distribution density

$$
\rho(\varphi)=\frac{1}{L} \sum_{k=1}^{M} \delta\left(\varphi-\varphi\left(p_{k}\right)\right), \quad \int_{\mathbf{C}} d \varphi \rho(\varphi)=\frac{M}{L}=\alpha .
$$

Now taking the thermodynamic limit of eqs.(2.15) and identifying $\rho$ with $\rho_{\mathrm{s}}$ we obtain the integral equation (2.14).

Due to the presence of the total charges it may be not clear why (2.15) is compatible with the principle of factorized scattering. However, taking into account formula (2.6) expressing the total charge $\mathbf{Q}_{r}$ as the sum of $\mathbf{q}_{r}\left(p_{k}\right)$ we see that eq.(2.15) is equivalent to eqs.(2.1), (2.2) if we bring the charge-dependent term on the r.h.s. In this form the factorization property becomes transparent. Indeed, the phase shift acquired by an individual excitation traveling around a circle of length $L$ is equal to the sum of pairwise phase shifts which arise due to its elastic scattering with the other $M-1$ excitations.

Concluding this section let us note that in the BMN limit, where the number $M$ of elementary excitations is kept finite, both $\mathbf{q}_{r}\left(p_{k}\right)$ and $\mathbf{Q}_{r}$ scale as $\mathbf{q}_{r}\left(p_{k}\right) \rightarrow L^{-r} \mathbf{q}_{r}\left(p_{k}\right)$ and $\mathbf{Q}_{r} \rightarrow L^{-r} \mathbf{Q}_{r}$ respectively. Therefore, the additional exponential term in the scattering matrix (2.2) appears to be $1 / L$ suppressed and drops out in the strict $L \rightarrow \infty$ limit. Therefore, in the BMN limit the resulting quantum Bethe equations coincide with the ones of the asymptotic Bethe ansatz and lead to the BMN energy formula (see [1]). However, the exponential term becomes relevant in considering $1 / L$ corrections, i.e. in the near-BMN limit. We will now turn our attention to this limit.

\section{Near-BMN Limit}

\subsection{Separated Mode Numbers}

The direct quantization of string theory in the BMN limit [2] is feasible since the complicated curved background $A d S_{5} \times S^{5}$ may be replaced by the much simpler ppwave background. In lightcone gauge it allows for an exact free field quantization, as first shown by Metsaev and Tseytlin [22]. The string predictions for the $\mathfrak{s u}(2)$ sector with $M$ impurities ${ }^{1}$ are immediately reproduced, by construction, on the gauge side if one uses the conjectured asymptotic long range spin chain Bethe ansatz of [11].

\footnotetext{
${ }^{1}$ In the condensed matter literature the elementary excitations of a ferromagnetic spin chain are certainly never denoted as "impurities" but rather as magnons. On the string side the nature of the elementary excitations at finite $L$ and $M$ is not known, but presumably related to some kind of "string bits".
} 
One keeps $M=2,3, \ldots$ finite, puts $L=J+M \sim J \rightarrow \infty$, and holds the BMN coupling $\lambda^{\prime}$ fixed:

$$
\lambda^{\prime}=\frac{\lambda}{J^{2}}
$$

Let us derive the famous BMN spectrum from our novel string Bethe ansatz (2.1), (2.2). The derivation is identical to the one for the long range spin chain for gauge theory [1]. One easily sees that the momenta scale like $p_{k} \sim 1 / J$ while the corresponding phase factors (2.3) scale like $\varphi\left(p_{k}\right) \sim J$. Inspection of the part of the discrete S-matrix (2.2) involving the phase function $\varphi\left(p_{k}\right)$ then shows that there is no scattering of the elementary excitations in the BMN limit. In other words, the excitations are too dilute to feel each other's presence, and behave like independent particles on a circle. It is an important and non-trivial property of our conjectured strong coupling string Bethe ansatz that the further terms, absent in the gauge theory ansatz, in the S-matrix (2.2), involving products of the excitation charges $\mathbf{q}_{r}\left(p_{k}\right)$, also do not lead to a scattering phase shift in the strict BMN limit, as will be shown shortly. This explains why in the latter case gauge and string theory agree to all orders in the coupling $\lambda^{\prime}$ (3.1). Accordingly the Bethe equations (2.1) simply become in both string and gauge theory

$$
e^{i p_{k} J}=1
$$

and are immediately solved by $p_{k}=\frac{2 \pi n_{k}}{J}$, where the $n_{k}$ are arbitrary integer mode numbers satisfying (from momentum conservation) $\sum_{k=1}^{M} n_{k}=0$. The energies $\Delta$ or anomalous dimensions $\Delta_{\mathrm{g}}$ are then found, without further work, in, respectively, string and gauge theory from (2.7), (2.8) as the eigenvalues of $\mathbf{E}(g), \mathbf{D}(g)$ :

$$
\Delta=\Delta_{\mathrm{g}}=J+\sum_{k=1}^{M} \sqrt{1+\lambda^{\prime} n_{k}^{2}} .
$$

The above argument for the absence of scattering appears to be invalid if some of the excitation numbers $n_{k}=n_{j}$ are coinciding, since then $\varphi\left(p_{k}\right) \rightarrow \varphi\left(p_{j}\right)$ in (2.2), and therefore the differences $\varphi\left(p_{k}\right)-\varphi\left(p_{j}\right)$ are no longer of order $\mathcal{O}(J)$. These cases require special analysis, see [6], and will be discussed in the next subsection. For the strict BMN limit one finds that this subtlety does not invalidate the final result (3.3).

The absence of scattering in the BMN limit shows, in a way, its comparative triviality. The situation gets much more interesting once curvature corrections to the pp-wave metric are taken into account. These should then correspond to $1 / J$ corrections to the BMN limit. This near-BMN limit has been investigated to leading order $\mathcal{O}(1 / J)$ on the string side in a number of papers, first for two excitations $(M=2)$ [23], 119, and, very recently, for three $(M=3)$ 20]. This required the 
inclusion of rather intricate and involved curvature corrections to the quantization procedure. Here we will show that our strong coupling Bethe ansatz is capable of reproducing, for the $\mathfrak{s u}(2)$ subsector, all these known results in a few lines of calculation $^{2}$. What is more, we are able to solve explicitly and without further work the generic $M$-excitation problem to $\mathcal{O}(1 / J)$ in generality, confer 3.8 below.

Let us then compute the leading correction to the large $J$ Bethe equation (3.2). One expands the excitation momenta $p_{k}$

$$
p_{k}=\frac{2 \pi n_{k}}{J}+\frac{p_{k}^{(2)}}{J^{2}}
$$

and works out the leading large $J$ behavior of the phases

$$
\varphi\left(p_{k}\right)=\frac{J}{2 \pi n_{k}} \sqrt{1+\lambda^{\prime} n_{k}^{2}}+\mathcal{O}\left(J^{0}\right)
$$

and the excitation charges

$$
\mathbf{q}_{r}\left(p_{k}\right)=\frac{2 \pi n_{k}}{J}\left[\frac{4 \pi}{\lambda^{\prime} J}\left(\sqrt{1+\lambda^{\prime} n_{k}^{2}}-1\right)\right]^{r-1}+\mathcal{O}\left(J^{-r-1}\right) .
$$

Then one expands the Bethe equations (2.1),(2.2) and verifies, after explicitly summing over the products of charges, that the scattering correction to the "free" BMN limit is indeed a $\mathcal{O}(1 / J)$ effect. One may then read off the momentum shift

$$
\frac{p_{k}^{(2)}}{2 \pi}=-\sum_{\substack{j=1 \\ j \neq k}}^{M} \frac{n_{k}^{2} \sqrt{1+\lambda^{\prime} n_{j}^{2}}+n_{j}^{2} \sqrt{1+\lambda^{\prime} n_{k}^{2}}}{n_{k}-n_{j}} .
$$

Let us note that the form of (3.7) manifestly leads to $\sum_{k=1}^{M} p_{k}^{(2)}=0$, as required by momentum conservation. Lastly, one expands the expression for the energy, (2.8) with (2.6), 2.5) to next-to-leading order, using the expansion (3.4). With the help of (3.7) one obtains the final, general energy for $M$ excitations, valid to order $\mathcal{O}(1 / J)$, and for non-coinciding mode numbers $n_{k}$ :

$$
\Delta=J+\sum_{k=1}^{M} \sqrt{1+\lambda^{\prime} n_{k}^{2}}-\frac{\lambda^{\prime}}{J} \sum_{\substack{k, j=1 \\ j \neq k}}^{M} \frac{n_{k}}{n_{k}-n_{j}}\left(n_{j}^{2}+n_{k}^{2} \sqrt{\frac{1+\lambda^{\prime} n_{j}^{2}}{1+\lambda^{\prime} n_{k}^{2}}}\right) .
$$

One easily verifies that this expression reproduces the near-BMN spectrum obtained by direct quantization for two $(M=2$, cf. [19]) and for three $(M=3$, cf. [20] eq.(4.10) on page 26) excitations in the $\mathfrak{s u}(2)$ sector.

${ }^{2}$ The present ansatz is not the only one that is capable of reproducing the near BMN results of [19], 20]. Another ansatz that also works was found by N. Beisert (unpublished). However, it appears to be very difficult to find a second ansatz that also reproduces the $\lambda^{\frac{1}{4}}$ strong coupling behavior of section 4 . 
The reader might find it interesting to compare this formula to the one obtained from the asymptotic gauge theory ansatz of [11]. Using exactly the same procedure as above we find

$$
\begin{aligned}
\Delta_{\mathrm{g}}=J+\sum_{k=1}^{M} \sqrt{1+\lambda^{\prime} n_{k}^{2}} & -\frac{\lambda^{\prime}}{J} \sum_{k=1}^{M} \frac{M n_{k}^{2}}{\sqrt{1+\lambda^{\prime} n_{k}^{2}}} \\
& -\frac{\lambda^{\prime}}{J} \sum_{\substack{k, j=1 \\
j \neq k}}^{M} \frac{2 n_{k}^{2} n_{j}}{n_{k}^{2}-n_{j}^{2}}\left(n_{j}+n_{k} \sqrt{\frac{1+\lambda^{\prime} n_{j}^{2}}{1+\lambda^{\prime} n_{k}^{2}}}\right) .
\end{aligned}
$$

This generalizes the formula for $M=2$, as first conjectured by N. Beisert in [8] (second reference) and subsequently derived in [11], to all $M$ (assuming separated mode numbers). The last sum in this expression could be formally further simplified, using antisymmetry under $j \leftrightarrow k$, by dropping the terms involving $n_{k}^{2} n_{j}^{2} /\left(n_{k}^{2}-n_{j}^{2}\right)$. We preferred the present form in order to manifestly exhibit the absence of poles at mode numbers related by $n_{j}=-n_{k}$. One can check, using momentum conservation, that (3.9) agrees with the string formula (3.8) for any $M$ up to two loops. At three loops they disagree for any $M$, as is to be expected by now.

\subsection{Confluent Mode Numbers}

Our general "generic" result (3.8) exhibits pole singularities if any two mode numbers are identical, and is therefore clearly nonsensical in this case. In fact, even the argument for the absence of scattering phase shifts in the strict BMN limit are flawed in the presence of confluences. As already pointed out in [6] the apparent inconsistency is resolved by the appearance of half-integer powers of $J$ in the expansion (3.4) of the momenta $p_{k}$. Let us denote by $\nu_{k}$ the multiplicities of momenta $p_{k, m_{k}}$ with leading identical mode number $n_{k}$, where the numbers $m_{k}=1, \ldots, \nu_{k}$ label the nearly degenerate momenta. Clearly one has

$$
\sum_{k=1}^{M^{\prime}} \nu_{k} n_{k}=0 \quad \text { and } \quad M=\sum_{k=1}^{M^{\prime}} \nu_{k},
$$

where $M^{\prime}$ is the number of distinct mode numbers. The refined expansion of the momenta reads ${ }^{3}$

$$
p_{k, m_{k}}=\frac{2 \pi n_{k}}{J}+\frac{p_{k, m_{k}}^{(1)}}{J^{\frac{3}{2}}}+\frac{p_{k, m_{k}}^{(2)}}{J^{2}}+\ldots
$$

\footnotetext{
${ }^{3}$ The behavior $J^{-\frac{3}{2}}$ is found by assuming a general correction $p_{k, m_{k}}=\frac{2 \pi n_{k}}{J}+\frac{p_{k, m_{k}}^{(1)}}{J^{a}}$ and matching powers in the expansions of both sides of the Bethe equations.
} 
Upon insertion into the Bethe equations (2.1), 2.2) one finds a non-linear system of equations, for each degenerate sector $k$ where $\nu_{k}>1$ [6]:

$$
p_{k, m_{k}}^{(1)}=-4 \pi^{2} n_{k}^{2} \sqrt{1+\lambda^{\prime} n_{k}^{2}} \sum_{\substack{\ell_{k}=1 \\ \ell_{k} \neq m_{k}}}^{\nu_{k}} \frac{2}{p_{k, m_{k}}^{(1)}-p_{k, \ell_{k}}^{(1)}} .
$$

Luckily this Stieltjes problem can be solved exactly [24, 25]. A nice method uses Baxter's $Q$-operator. For a recent discussion of a similar (but different) equation system of this type see [26. We find the solution

$$
\left(p_{k, m_{k}}^{(1)}\right)^{2}=-4 \pi^{2} n_{k}^{2} \sqrt{1+\lambda^{\prime} n_{k}^{2}} u_{\nu_{k}, m_{k}}^{2},
$$

where the $u_{\nu_{k}, m_{k}}$ are the $\nu_{k}$ roots of the Hermite polynomials

$$
Q_{\nu_{k}}(u)=2^{\frac{\nu_{k}}{2}} H_{\nu_{k}}\left(\frac{u}{\sqrt{2}}\right)=\prod_{m_{k}=1}^{\nu_{k}}\left(u-u_{\nu_{k}, m_{k}}\right)
$$

satisfying the differential equation $Q^{\prime \prime}(u)-u Q^{\prime}(u)+\nu_{k} Q(u)=0$. We thus see that the degeneracy of the momenta $p_{k, m_{k}}$ is lifted by $\nu_{k}$ distinct, purely imaginary shifts into the complex plane, proving the suppression of scattering in the strict BMN limit. One also checks that these shifts do not lead to unwanted $J^{-\frac{1}{2}}$ corrections to the energies. In order to compute the $1 / J$ correction to the energy in the confluent case, we first find an expansion of the dimension formula (2.8) up to the order $1 / J$ :

$$
\begin{aligned}
\Delta=J & +\sum_{k=1}^{M^{\prime}} \nu_{k} \sqrt{1+\lambda^{\prime} n_{k}^{2}} \\
& +\frac{\lambda^{\prime}}{J} \sum_{k=1}^{M^{\prime}} \sum_{m_{k}=1}^{\nu_{k}} \frac{\left(p_{k, m_{k}}^{(1)}\right)^{2}}{8 \pi^{2}\left(1+\lambda^{\prime} n_{k}^{2}\right)^{3 / 2}}+\frac{\lambda^{\prime}}{J} \sum_{k=1}^{M^{\prime}} \sum_{m_{k}=1}^{\nu_{k}} \frac{n_{k} p_{k, m_{k}}^{(2)}}{2 \pi \sqrt{1+\lambda^{\prime} n_{k}^{2}}}
\end{aligned}
$$

The sum over $m_{k}$ of $\left(p_{k, m_{k}}^{(1)}\right)^{2}$ on the second line of (3.15) can be easily computed by using (3.13) and the following formula for the roots of Hermite polynomials

$$
\sum_{m_{k}=1}^{\nu_{k}}\left(u_{\nu_{k}, m_{k}}\right)^{2}=\nu_{k}\left(\nu_{k}-1\right)
$$

Thus, it is sufficient to find the sum over $m_{k}$ of $p_{k, m_{k}}^{(2)}$ to compute the $1 / J$ correction to the energy. Because of the summation over $m_{k}$ the contribution of momenta with the same mode number $n_{k}$ drops out, and the Bethe equations can be easily solved with the following result

$$
\sum_{m_{k}=1}^{\nu_{k}} \frac{p_{k, m_{k}}^{(2)}}{2 \pi}=-\sum_{\substack{j=1 \\ j \neq k}}^{M^{\prime}} \frac{\nu_{k} \nu_{j}}{n_{k}-n_{j}}\left(n_{j}^{2} \sqrt{1+\lambda^{\prime} n_{k}^{2}}+n_{k}^{2} \sqrt{1+\lambda^{\prime} n_{j}^{2}}\right) .
$$


Combining all the expressions together, we finally find

$$
\begin{aligned}
\Delta=J+\sum_{k=1}^{M^{\prime}} \nu_{k} \sqrt{1+\lambda^{\prime} n_{k}^{2}} & -\frac{\lambda^{\prime}}{J} \sum_{k=1}^{M^{\prime}} \frac{\nu_{k}\left(\nu_{k}-1\right) n_{k}^{2}}{2\left(1+\lambda^{\prime} n_{k}^{2}\right)} \\
& -\frac{\lambda^{\prime}}{J} \sum_{\substack{k, j=1 \\
j \neq k}}^{M^{\prime}} \frac{\nu_{k} \nu_{j} n_{k}}{n_{k}-n_{j}}\left(n_{j}^{2}+n_{k}^{2} \sqrt{\frac{1+\lambda^{\prime} n_{j}^{2}}{1+\lambda^{\prime} n_{k}^{2}}}\right) .
\end{aligned}
$$

In the simplest case where $M=3$, with $\nu_{1}=2, \nu_{2}=1$ and $n_{1}:=n, n_{2}=-2 n$ we obtain using (3.18)

$$
\begin{aligned}
\Delta= & J+2 \sqrt{1+\lambda^{\prime} n^{2}}+\sqrt{1+\lambda^{\prime} 4 n^{2}} \\
& -\frac{\lambda^{\prime} n^{2}}{J}\left[\frac{6+8 \lambda^{\prime} n^{2}}{\sqrt{1+\lambda^{\prime} n^{2}} \sqrt{1+\lambda^{\prime} 4 n^{2}}}+\frac{5+4 \lambda^{\prime} n^{2}}{1+\lambda^{\prime} n^{2}}\right] .
\end{aligned}
$$

It agrees precisely with the result of direct quantization in [20], eq.(4.21) on page 30 .

For the reader's convenience we also obtained the analogous formula following from the asymptotic gauge theory ansatz of [1]:

$$
\begin{aligned}
\Delta_{\mathrm{g}}=J & +\sum_{k=1}^{M^{\prime}} \nu_{k} \sqrt{1+\lambda^{\prime} n_{k}^{2}}-\frac{\lambda^{\prime}}{J} \sum_{k=1}^{M^{\prime}} \frac{\nu_{k}\left(\nu_{k}-1\right) n_{k}^{2}}{2\left(1+\lambda^{\prime} n_{k}^{2}\right)} \\
& -\frac{\lambda^{\prime}}{J} \sum_{k=1}^{M^{\prime}} \frac{M \nu_{k} n_{k}^{2}}{\sqrt{1+\lambda^{\prime} n_{k}^{2}}}-\frac{\lambda^{\prime}}{J} \sum_{\substack{k, j=1 \\
j \neq k}}^{M^{\prime}} \frac{2 \nu_{k} \nu_{j} n_{k}^{2} n_{j}}{n_{k}^{2}-n_{j}^{2}}\left(n_{j}+n_{k} \sqrt{\frac{1+\lambda^{\prime} n_{j}^{2}}{1+\lambda^{\prime} n_{k}^{2}}}\right) .
\end{aligned}
$$

One easily checks that it also agrees with the string formula (3.19) up to, but not beyond, two loops. One can also see that at one loop, our formula coincides with the one obtained in [6]. In the simplest three-impurity case we obtain using the formula

$$
\begin{aligned}
\Delta_{\mathrm{g}}= & J+2 \sqrt{1+\lambda^{\prime} n^{2}}+\sqrt{1+\lambda^{\prime} 4 n^{2}} \\
& -\frac{\lambda^{\prime} n^{2}}{J}\left[\frac{1}{1+\lambda^{\prime} n^{2}}+\frac{6}{\sqrt{1+\lambda^{\prime} n^{2}}}+\frac{12}{\sqrt{1+\lambda^{\prime} 4 n^{2}}}-\frac{8}{\sqrt{1+\lambda^{\prime} n^{2}} \sqrt{1+\lambda^{\prime} 4 n^{2}}}\right] .
\end{aligned}
$$

\section{Strong Coupling Limit}

The quantum Bethe equations allow us to analyze the strong coupling limit $\lambda \rightarrow \infty$ with $L \ll \sqrt[4]{\lambda}$. In this case we should expect to find the famous $\sqrt[4]{\lambda}$ behavior of operators dual to massive string modes.

We start by considering the simplest case of two excitations: $M=2$. Assuming $p>0$ in the large $g$ limit the charge densities turn into

$$
\mathbf{q}_{r}(p) \rightarrow g^{-r+1} \frac{2 \sin \left(\frac{1}{2}(r-1) p\right)}{(r-1)}(\sqrt{2})^{r-1}
$$


and the sum over $n$ in the scattering matrix (2.2) can be taken explicitly. Then eq.(2.1) acquires the form

$$
\exp \left(i L p-8 i \sqrt{2} g \cos \left(\frac{1}{2} p\right) \log \left(\cos \left(\frac{1}{2} p\right)\right)\right)=1
$$

where we have taken into account that the phase function $\varphi(p) \rightarrow \infty$ in the large $g$ limit. This equation can be easily solved assuming that the momentum $p$ has an expansion

$$
p=\frac{p_{0}}{\sqrt{g}}+\frac{p_{1}}{g}+\ldots
$$

Substituting the expansion into (4.2), we get the leading contribution

$$
\exp \left(i \sqrt{2} p_{0}^{2}\right)=1
$$

and, therefore,

$$
p_{0}=2^{\frac{1}{4}} \sqrt{n \pi} .
$$

We want to emphasize that $p_{0}$ is completely determined by the exponential term in the scattering matrix (2.2). Note in particular that the term $L p \sim \frac{L p_{0}}{\sqrt{g}}$ in the l.h.s. of (4.2) does not contribute into the leading asymptotics due to our restriction on $L$ : $L \ll \sqrt{g}$.

The conformal dimension $\Delta$ of the operator is obtained from eq.(2.8), and its leading large $\lambda$ asymptotics is given by

$$
\Delta=2\left(n^{2} \lambda\right)^{\frac{1}{4}}
$$

This is exactly what one expects to find for dimensions of operators dual to massive string modes at level $n$ with masses $m^{2}=4 n \sqrt{\lambda}$ [27].

Let us now understand what happens for a generic case of $M$ excitations. To this end we have to consider the sum

$$
\chi\left(p_{k}, p_{j}\right)=\sum_{r=0}^{\infty}\left(\frac{g^{2}}{2}\right)^{r+2}\left(\mathbf{q}_{r+3}\left(p_{k}\right) \mathbf{q}_{r+2}\left(p_{j}\right)-\mathbf{q}_{r+2}\left(p_{k}\right) \mathbf{q}_{r+3}\left(p_{j}\right)\right) .
$$

The roots $p_{k}$ (generically complex) obey the conservation law $\sum_{k=1}^{M} p_{k}=0$ and can be grouped into two sets: $p_{k}^{+}$with $\operatorname{Re} p_{k}>0, k=1, \ldots, m$ and $p_{k}^{-}$with $\operatorname{Re} p_{k}<0$, $k=m+1, \ldots, M$. Once again we expect $p_{k}$ to scale as in (4.3). It is therefore convenient to first rescale $p \rightarrow \frac{1}{\sqrt{g}} p$ and then consider the $\operatorname{limit}^{4} g \rightarrow \infty$. Note that

\footnotetext{
${ }^{4}$ Concerning eq. $(4.5)$, one should first perform the sum and only then take the limit with rescaled momenta substituted.
} 
the definition of the charges $\mathbf{q}_{r}\left(p_{k}\right)$ requires some care and the correct prescription for picking up the sign is

$$
\frac{\sqrt{\sin ^{2}\left(\frac{1}{2} p\right)}}{\sin \left(\frac{1}{2} p\right)}=\operatorname{sign} \operatorname{Re} p
$$

Now taking the strong coupling limit we find that the scaling dimension is expressed via rescaled momenta as

$$
\Delta=\left(\frac{\lambda}{2 \pi^{2}}\right)^{\frac{1}{4}}\left(\sum_{k=1}^{m} p_{k}^{+}-\sum_{k=m+1}^{M} p_{k}^{-}\right)
$$

while the Bethe ansatz equations (2.15) turn into

$$
\sum_{j=1}^{m} \chi_{k j}^{++}+\sum_{j=m+1}^{M} \chi_{k j}^{+-}=-\pi n_{k}
$$

where $n_{k} \geq 0$ are mode numbers. Here we have also introduced the concise notation $\chi_{k j}^{ \pm \pm}=\chi\left(p_{k}^{ \pm}, p_{j}^{ \pm}\right)$to keep track of the signs. There are also equations involving $\chi_{k j}^{--}$ and $\chi_{k j}^{-+}$but they are of no relevance to us.

Explicit computation of the leading contribution of $\chi_{k j}^{+-}$gives

$$
\chi_{k j}^{+-}=\frac{1}{\sqrt{2}} p_{k}^{+} p_{j}^{-},
$$

while the formula for $\chi_{k j}^{++}$appears to be rather involved. Fortunately, as we show below, we do not need it here.

Let us sum eqs.(4.7) over $k$ :

$$
\sum_{k=1}^{m} \sum_{j=1}^{m} \chi_{k j}^{++}+\sum_{k=1}^{m} \sum_{j=m+1}^{M} \chi_{k j}^{+-}=-\pi \sum_{k=1}^{m} n_{k} .
$$

Since $\chi_{k j}^{++}=-\chi_{j k}^{++}$the first sum vanishes and we are left with

$$
\sum_{k=1}^{m} \sum_{j=m+1}^{M} \chi_{k j}^{+-}=\frac{1}{\sqrt{2}}\left(\sum_{k=1}^{m} p_{k}^{+}\right)\left(\sum_{j=m+1}^{M} p_{j}^{-}\right)=-\frac{1}{\sqrt{2}}\left(\sum_{k=1}^{m} p_{k}^{+}\right)^{2}=-\pi \sum_{k=1}^{m} n_{k},
$$

where we have used eq.(4.8) and the momentum conservation law. Therefore, we get

$$
\sum_{k=1}^{m} p_{k}^{+}=-\sum_{k=m+1}^{M} p_{k}^{-}=2^{\frac{1}{4}} \sqrt{\pi \sum_{k=1}^{m} n_{k}}
$$

With this formula at hand the scaling dimension eq.(4.6) now reads

$$
\Delta=2\left(\left(\sum_{k=1}^{m} n_{k}\right)^{2} \lambda\right)^{\frac{1}{4}} .
$$


This formula generalizes eq.(4.4) to the case of arbitrary number of elementary excitations and shows that the corresponding "gauge theory" operators are dual to string modes with masses: $m^{2}=4 n \sqrt{\lambda}$, where the level $n$ is now determined by the mode numbers of roots with a positive real part: $n=\sum_{k=1}^{m} n_{k}$. One can think of excitations $p_{k}^{+}$and $p_{k}^{-}$as representing right- and left-moving string modes, and eq.(4.9) as the level matching condition.

The fact that we do not get any restrictions on $n_{k}$ means that the quantum Bethe ansatz equations may only describe strong coupling limit of long operators with large $L$. The simplest way to see that is to notice that the Konishi operator is dual to the lightest massive string mode and, therefore, its anomalous dimension should have $n=1$ in eq.(4.4). Since for $L=4$ and $M=2$ the only unprotected operator is a Konishi descendant, quantum Bethe equations valid for all values of $L$ would restrict the level $n$ to be $1 .^{5}$

\section{Wrapping Speculations}

As we have already pointed out the string Bethe ansatz (2.1) differs from the all-loop asymptotic Bethe ansatz of [11] by the exponential term in the S-matrix (2.2). This term captures the essential dynamics of quantum strings in the large $g, L$ limit. Note that we still need to assume $L \gg 1$, but we do not (unlike in the BMN and spinning strings limits) require $L \sim g$. However, hypothetically assuming the string ansatz to be valid for all values of $g$ and $L$ as is, it leads to disagreement with perturbative gauge theory. In particular, the known three-loop scaling dimension of the Konishi operator [8] is not reproduced by the string Bethe ansatz. In fact, the ansatz looks rather different from all the standard Bethe ansätze for quantum spin chains.

It was proposed in [10, 11] that disagreement between gauge and string theory predictions might be due to neglecting the wrapping interactions in gauge theory. At the $k$-th order of perturbation theory the dilatation operator is given by the sum of multi-spin interactions, each of them involving up to $k+1$ neighboring sites of the spin chain. Therefore, with increasing $k$ the non-locality of the interactions grows until it encompasses, at $k=L-1$, all lattice sites of the chain. Starting at this loop order the interactions wrap around the chain and the assumptions of the asymptotic Bethe ansatz break down. Since the string predictions are derived assuming that both $g$ and $L$ are large, the comparison with gauge theory requires resummation of the gauge theoretic perturbative expansion, i.e. inclusion of the wrapping interactions [10, 11]. At present their structure is unknown. Moreover, the very notion of integrability becomes more subtle because the locality of the spin chain model is lost. In this situation one can try to get some insight on the possible structure of the full, nonasymptotic gauge Bethe ansatz from our string theory ansatz.

\footnotetext{
${ }^{5}$ We thank N. Beisert for an important discussion on this point.
} 
Looking at the S-matrix (2.2) we see that the only explicit dependence on the coupling constant $g$ appears in the sum entering the exponential terms. All other dependence is hidden in the phase function and charges. The simplest guess for modifying the string scattering matrix so that it becomes compatible with gauge theory seems to consist in replacing the coefficients $\left(\frac{g^{2}}{2}\right)^{r+2}$ by more general functions $c_{r}(g, L)$ which depend on both $g$ and $L$. Thus, we are led to consider the Bethe ansatz (2.1) with the following S-matrix

$$
\begin{aligned}
S\left(p_{k}, p_{j}\right) & =\frac{\varphi\left(p_{k}\right)-\varphi\left(p_{j}\right)+i}{\varphi\left(p_{k}\right)-\varphi\left(p_{j}\right)-i} \times \\
& \times \exp \left(2 i \sum_{r=0}^{\infty} c_{r}(g, L)\left(\mathbf{q}_{r+2}\left(p_{k}\right) \mathbf{q}_{r+3}\left(p_{j}\right)-\mathbf{q}_{r+3}\left(p_{k}\right) \mathbf{q}_{r+2}\left(p_{j}\right)\right)\right) .
\end{aligned}
$$

Once again we assume that the phase function $\varphi(p)$, the excitation charges $\mathbf{q}_{n}(p)$ and the total charges $\mathbf{Q}_{n}$ are literally the same as appeared in the asymptotic Bethe ansatz, and given by eqs.(2.3), (2.4) and (2.6). The exponential term in the S-matrix depends now on an infinite set of functions $c_{r}(g, L)$. From the gauge theory point of view the role of this term might hopefully account for the wrapping interactions, and, in the following we will conjecturally call it the "wrapping term".

We do not have much to say about the explicit form of the functions $c_{r}$; however, in order to be consistent with both perturbative gauge theory and the known string theory results these functions have to exhibit the following properties: ${ }^{6}$

- $c_{r}(g, L) \rightarrow 0$ in the asymptotic limit, $L \rightarrow \infty$ and $g$ is held finite;

- $c_{r}(g, L) \sim \mathcal{O}\left(g^{2(L-1)}\right)$ in the perturbative gauge theory, $g \ll 1$ and $L$ is finite;

- $c_{r}(g, L) \rightarrow\left(\frac{g^{2}}{2}\right)^{r+2}$ in the limit $L, g \rightarrow \infty$ and $\frac{g}{L}$ is held finite;

- $c_{r}(g, L) \rightarrow\left(\frac{g^{2}}{2}\right)^{r+2}$ in the strong coupling limit, $g \rightarrow \infty$ and $1 \ll L \ll \sqrt{g}$.

Let us now motivate these asymptotic properties of $c_{r}$. First of all, we demand the vanishing of the $c_{r}$ in the asymptotic limit to guarantee that the S-matrix (5.1) reduces to the S-matrix of the gauge theory asymptotic Bethe ansatz. The second property of $c_{r}$ ensures that the wrapping interactions do not show up at least up to the $L$-th order of perturbation theory, which is another important feature of gauge

\footnotetext{
${ }^{6} \mathrm{It}$ is easy to find an example of a function with such properties. For instance one can take (cf. [11)

$$
c_{r}(g, L)=\left(\frac{g^{2}}{2}\right)^{r+2} \tanh ^{2(L-r-3)}(g) \quad \text { or } \quad c_{r}(g, L)=\left(1+\frac{g^{2}}{2}\right)^{r+2} \frac{g^{2(L-1)}}{\left(1+g^{2}\right)^{(L-1)}} .
$$
}


theory. The third and the fourth properties are needed to recover our string Bethe ansatz at strong coupling and, in particular, to obtain the $\sqrt[4]{\lambda}$ asymptotics of scaling dimensions. Thus, the functions $c_{r}$ might allow to interpolate smoothly between gauge and string theories, and, for this reason, it is natural to term the equations (2.1), (5.1) "interpolating Bethe ansatz".

One could hope that the explicit form of the functions $c_{r}$ might be determined by the exact quantization of strings on $A d S_{5} \times S^{5}$. Moreover, the Bethe equations themselves provide a very tight restriction on a possible quantization ansatz. On the other hand, we expect that the interpolating Bethe ansatz might correctly account for wrapping interactions in gauge theory, and, therefore, encode the gauge theory perturbative spectrum. With wrapping interactions turned off the equations (2.1), (2.15) reduce to the ones of the asymptotic Bethe ansatz and, therefore, immediately reproduce all known results in perturbative gauge theory. Thus, the true test of the validity of the interpolating Bethe ansatz in gauge theory essentially relies on the currently unknown structure of the wrapping interactions.

The conjecture of the interpolating Bethe ansatz implies, quite remarkably, that from the gauge theory point of view, the $\sqrt[4]{\lambda}$ asymptotics is entirely due to the wrapping term. For the near-BMN limit it leads to an important modification of the result obtained by the asymptotic Bethe ansatz.

To conclude this section let us note that our proposal of the interpolating Bethe ansatz is just a first step towards an exact quantization of strings on $A d S_{5} \times S^{5}$ in the $\mathfrak{s u}(2)$ subsector. It is important to find internal consistency conditions for eqs. (2.1), (2.2). This might help to understand if other integrable systems, in particular spin chain models, can be described by similar equations. In this respect the Inozemtsev long-range spin chain seems to be a natural candidate for investigating this point.

\section{Summary and Conclusions}

In this paper we proposed a novel set of Bethe ansatz equations. These equations can be thought of as encoding the quantum spectrum of superstring theory on $\operatorname{Ad} S_{5} \times$ $S^{5}$ at large tension and restricted to the large charge states from the closed $\mathfrak{s u}(2)$ subsector. Important evidence for such an interpretation comes from our study of the thermodynamic, near-BMN, and strong coupling limits.

In the thermodynamic limit (2.1), (2.2) reproduce the energies of classical spinning strings, and in the near-BMN limit they give the $1 / J$ correction to the BMN energy formula which agrees and extends the results obtained by quantizing string theory in the near plane-wave limit. Finally, in the strong coupling limit they reproduce the correct $\sqrt[4]{\lambda}$ behavior of anomalous dimensions. Thus, these equations are compatible with our current knowledge of the string theory spectrum on $A d S_{5} \times S^{5}$. 
It would be interesting to find proper generalizations of our $\mathfrak{s u}(2)$ Bethe equations to other closed subsectors.

We also suggested a "minimal" deformation of our string Bethe equations which might hopefully describe a smooth interpolation between perturbative gauge theory and string theory at large tension. These interpolating Bethe equations involve an infinite set of functions $c_{r}(g, L)$ known only in certain asymptotic regimes. We speculate that these functions might capture the dynamics of gauge theory wrapping interactions and lead to the final agreement of gauge and string predictions.

Another, more disappointing scenario would be that free strings on $A d S_{5} \times S^{5}$ and planar $\mathcal{N}=4$ Super Yang-Mills theory are described by similar, but different integrable systems. If true, this would disprove the AdS/CFT conjecture [1].

There are several immediate questions one can ask. Here we computed the $1 / J$ correction to the BMN energy formula for the general case of $M$ impurities. The results of exact quantization around a plane-wave background are known only for the states with two and three impurities [19, 20]. It would be interesting to generalize the calculation of [19] to the case of four (and more) impurities to check the predictions of the string Bethe ansatz.

Note also that the functions $c_{r}$ governing the interpolating Bethe ansatz can be partially fixed from string theory by analyzing $1 / J$ and $1 / \sqrt{\lambda}$ corrections. There are several ways to proceed. One is to determine $1 / J^{2}$ correction to the BMN energy formula by using the techniques developed in [19]. A step in this direction has been recently made in [28], although many details still have to be clarified. Another way is to compute the $1 / J$ correction to the energy of the two-spin folded string along the lines of [4]. One can also develop the quantization of the light-cone Hamiltonian of string theory on $A d S_{5} \times S^{5}$ [29] to compute $1 / \sqrt{\lambda}$ corrections to masses of string states in the large tension limit. All these computations would provide further stringent tests of our interpolating Bethe ansatz conjecture.

Of course, one of the most ambitious problems is to derive integrability along with the corresponding complete quantum Bethe ansatz by exactly quantizing strings on $A d S_{5} \times S^{5}$.

\section{Acknowledgments}

We are very grateful to Niklas Beisert and Arkady Tseytlin for many useful discussions and helpful comments. S. F. wishes to thank the Max-Planck-Institut für Gravitationsphysik for the warm hospitality. The work of G. A. was supported in part by the European Commission RTN programme HPRN-CT-2000-00131 and by RFBI grant N02-01-00695. The work of S. F. was supported in part by the 2004 Crouse Award. 


\section{References}

[1] J. M. Maldacena, "The large $N$ limit of superconformal field theories and supergravity," Adv. Theor. Math. Phys. 2 (1998) 231 [Int. J. Theor. Phys. 38 (1999) 1113], hep-th/9711200.

[2] D. Berenstein, J. M. Maldacena and H. Nastase, "Strings in flat space and pp waves from $N=4$ super Yang Mills," JHEP 0204 (2002) 013, hep-th/0202021.

[3] S. S. Gubser, I. R. Klebanov and A. M. Polyakov, "A semi-classical limit of the gauge/string correspondence," Nucl. Phys. B 636 (2002) 99, hep-th/0204051.

[4] S. Frolov and A. A. Tseytlin, "Semiclassical quantization of rotating superstring in $A d S_{5} \times S^{5}$," JHEP 0206 (2002) 007, hep-th/0204226; "Multi-spin string solutions in $A d S_{5} \times S^{5}$," Nucl. Phys. B 668 (2003) 77, hep-th/0304255; "Quantizing three-spin string solution in $A d S_{5} \times S^{5}$," JHEP 0307 (2003) 016, hep-th/0306130; "Rotating string solutions: AdS/CFT duality in non-supersymmetric sectors," Phys. Lett. B 570 (2003) 96, hep-th/0306143.

[5] G. Arutyunov, S. Frolov, J. Russo and A. A. Tseytlin, "Spinning strings in $A d S_{5} \times S^{5}$ and integrable systems," Nucl. Phys. B 671, 3 (2003), hep-th/0307191;

G. Arutyunov, J. Russo and A. A. Tseytlin, "Spinning strings in $A d S_{5} \times S^{5}: N e w$ integrable system relations," Phys. Rev. D 69 (2004) 086009, hep-th/0311004.

[6] J. A. Minahan and K. Zarembo, "The Bethe-ansatz for $N=4$ super Yang-Mills," JHEP 0303, (2003) 013, hep-th/0212208.

[7] N. Beisert, "The complete one-loop dilatation operator of $N=4$ super Yang-Mills theory," hep-th/0307015; N. Beisert and M. Staudacher, "The N=4SYM integrable super spin chain," hep-th/0307042.

[8] N. Beisert, C. Kristjansen and M. Staudacher, "The dilatation operator of $N=4$ super Yang-Mills theory," Nucl. Phys. B 664 (2003) 131, hep-th/0303060; N. Beisert, "Higher loops, integrability and the near BMN limit," hep-th/0308074; N. Beisert, "The su(2|3) dynamic spin chain," Nucl. Phys. B 682 (2004) 487, hep-th/0310252.

[9] V. M. Braun, S. E. Derkachov and A. N. Manashov, "Integrability of three-particle evolution equations in QCD," Phys. Rev. Lett. 81 (1998) 2020 hep-ph/9805225. V. M. Braun, S. E. Derkachov, G. P. Korchemsky and A. N. Manashov, "Baryon distribution amplitudes in QCD," Nucl. Phys. B 553 (1999) 355 hep-ph/9902375. A. V. Belitsky, "Renormalization of twist-three operators and integrable lattice models," Nucl. Phys. B 574 (2000) 407 hep-ph/9907420.

[10] D. Serban and M. Staudacher, "Planar $N=4$ gauge theory and the Inozemtsev long range spin chain,” JHEP 0406 (2004) 001, hep-th/0401057.

[11] N. Beisert, V. Dippel and M. Staudacher, "A novel long range spin chain and planar $N=4$ super Yang-Mills," hep-th/0405001. 
[12] N. Beisert, J. A. Minahan, M. Staudacher and K. Zarembo, "Stringing spins and spinning strings," JHEP 0309 (2003) 010, hep-th/0306139; N. Beisert, S. Frolov, M. Staudacher and A. A. Tseytlin, "Precision spectroscopy of AdS/CFT," hep-th/0308117.

[13] G. Arutyunov and M. Staudacher, "Matching higher conserved charges for strings and spins," JHEP 0403 (2004) 004, hep-th/0310182; "Two-loop commuting charges and the string / gauge duality," hep-th/0403077.

[14] V. A. Kazakov, A. Marshakov, J. A. Minahan and K. Zarembo, "Classical / quantum integrability in AdS/CFT,” JHEP 0405 (2004) 024 hep-th/0402207.

[15] J. Engquist, J. A. Minahan and K. Zarembo, "Yang-Mills duals for semiclassical strings on $A d S_{5} \times S^{5}$ ", hep-th/0310188; J. Engquist, "Higher conserved charges and integrability for spinning strings in $A d S_{5} \times S^{5}$ ”, JHEP 0404 (2004) 002,

hep-th/0402092; M. Smedback, "Pulsating Strings On AdS $S_{5} \times S^{5}$," hep-th/0405102; L. Freyhult, "Bethe ansatz and fluctuations in SU(3) Yang-Mills operators", JHEP 0406 (2004) 010, hep-th/0405167; J. A. Minahan, "Higher loops beyond the SU(2) sector," hep-th/0405243.

[16] M. Kruczenski, "Spin chains and string theory," hep-th/0311203; M. Kruczenski, A. V. Ryzhov and A. A. Tseytlin, "Large spin limit of $A d S_{5} \times S^{5}$ string theory and low energy expansion of ferromagnetic spin chains," hep-th/0403120; H. Dimov and R. C. Rashkov, "A note on spin chain / string duality," hep-th/0403121.

R. Hernandez and E. Lopez, "The SU(3) spin chain sigma model and string theory," JHEP 0404, 052 (2004), hep-th/0403139. B. . J. Stefanski and A. A. Tseytlin, "Large spin limits of AdS/CFT and generalized Landau-Lifshitz equations," JHEP 0405, 042 (2004) hep-th/0404133; A. V. Ryzhov and A. A. Tseytlin, "Towards the exact dilatation operator of $N=4$ super Yang-Mills theory," hep-th/0404215; M. Kruczenski and A. A. Tseytlin, "Semiclassical relativistic strings in $S^{5}$ and long coherent operators in $N=4 S Y M$ theory," hep-th/0406189.

[17] A. Khan and A. L. Larsen, "Spinning pulsating string solitons in $A d S_{5} \times S^{5}$," Phys. Rev. D 69, 026001 (2004), hep-th/0310019; A. L. Larsen and A. Khan, "Novel explicit multi spin string solitons in AdS(5)," Nucl. Phys. B 686, 75 (2004) hep-th/0312184; C. Kristjansen, "Three-spin strings on $A d S_{5} \times S^{5}$ from $N=4$ SYM," Phys. Lett. B 586 (2004) 106, hep-th/0402033; C. Kristjansen and T. Mansson, "The Circular, Elliptic Three Spin String from the SU(3) Spin Chain," hep-th/0406176.

[18] J. G. Russo, "Anomalous dimensions in gauge theories from rotating strings in $A d S_{5} \times S^{5}$," JHEP 0206 (2002) 038, hep-th/0205244; J. A. Minahan, "Circular semiclassical string solutions on $A d S_{5} \times S^{5}$," Nucl. Phys. B 648 (2003) 203 hep-th/0209047; A. V. Belitsky, A. S. Gorsky and G. P. Korchemsky, "Gauge / string duality for QCD conformal operators," Nucl. Phys. B 667 (2003) 3, hep-th/0304028; A. Mikhailov, "Speeding strings," JHEP 0312, 058 (2003), hep-th/0311019; 
A. Mikhailov, "Slow evolution of nearly-degenerate extremal surfaces," hep-th/0402067. A. Mikhailov, "Supersymmetric null-surfaces," hep-th/0404173; S. Ryang, "Folded three-spin string solutions in $A d S_{5} \times S^{5}$," JHEP 0404, 053 (2004), hep-th/0403180; H. Dimov and R. C. Rashkov, "Generalized pulsating strings," JHEP 0405, 068 (2004), hep-th/0404012; A. Agarwal and S. G. Rajeev, "The dilatation operator of $N=4 S Y M$ and classical limits of spin chains and matrix models," hep-th/0405116; M. Alishahiha, A. E. Mosaffa and H. Yavartanoo, "Multi-spin string solutions in AdS black hole and confining backgrounds," Nucl. Phys. B 686 (2004) 53 hep-th/0402007. F. Bigazzi, A. L. Cotrone and L. Martucci, "Semiclassical spinning strings and confining gauge theories," hep-th/0403261.

[19] C. G. Callan, H. K. Lee, T. McLoughlin, J. H. Schwarz, I. Swanson and X. Wu, "Quantizing string theory in $A d S_{5} \times S^{5}$ : Beyond the pp-wave," Nucl. Phys. B 673 (2003) 3, hep-th/0307032; C. G. Callan, T. McLoughlin and I. Swanson, "Holography beyond the Penrose limit," hep-th/0404007.

[20] C. G. Callan, T. McLoughlin and I. Swanson, "Higher impurity AdS/CFT correspondence in the near-BMN limit," hep-th/0405153.

[21] V. I. Inozemtsev, "Integrable Heisenberg-van Vleck chains with variable range exchange," Phys. Part. Nucl. 34 (2003) 166 [Fiz. Elem. Chast. Atom. Yadra 34 (2003) 332], hep-th/0201001.

[22] R. R. Metsaev, "Type IIB Green-Schwarz superstring in plane wave Ramond-Ramond background," Nucl. Phys. B 625 (2002) 70, hep-th/0112044; R. R. Metsaev and A. A. Tseytlin, "Exactly solvable model of superstring in plane wave Ramond-Ramond background," Phys. Rev. D 65 (2002) 126004, hep-th/0202109.

[23] A. Parnachev and A. V. Ryzhov, "Strings in the near plane wave background and AdS/CFT," JHEP 0210 (2002) 066, hep-th/0208010.

[24] T. J. Stieltjes, "Sur Quelques Theoremes d'Algebre," Oeuvres Completes, vol. 1, p 440, Noordhoff, Groningen, The Netherlands (1914).

[25] B. S. Shastry, A. Dhar, Solution of a Generalized Stieltjes Problem, cond-mat/0101464.

[26] M. Lübcke and K. Zarembo, "Finite-size corrections to anomalous dimensions in $N$ = 4 SYM theory," JHEP 0405 (2004) 049, hep-th/0405055.

[27] S. S. Gubser, I. R. Klebanov and A. M. Polyakov, "Gauge theory correlators from non-critical string theory," Phys. Lett. B 428 (1998) 105, hep-th/9802109.

[28] I. Swanson, "On the integrability of string theory in $A d S_{5} \times S^{5}$," hep-th/0405172.

[29] R. R. Metsaev and A. A. Tseytlin, Type IIB superstring action in $A d S_{5} \times S^{5}$ background," Nucl. Phys. B 533 (1998) 109, hep-th/9805028; R. R. Metsaev, C. B. Thorn and A. A. Tseytlin, "Light-cone superstring in AdS space-time," Nucl. Phys. B 596 (2001) 151, hep-th/0009171. 